\title{
General Psychiatry Role of biological rhythm dysfunction in the development and management of bipolar disorders: a review
}

\author{
Yiming Chen, ${ }^{1}$ Wu Hong, ${ }^{1}$ Yiru Fang ${ }^{1,2,3}$
}

To cite: Chen Y, Hong W, Fang Y. Role of biological rhythm dysfunction in the development and management of bipolar disorders: a review. General Psychiatry 2020;33:e100127. doi:10.1136/ gpsych-2019-100127

Received 13 August 2019 Revised 24 September 2019 Accepted 28 November 2019

Check for updates

(c) Author(s) (or their employer(s)) 2020. Re-use permitted under CC BY-NC. No commercial re-use. See rights and permissions. Published by BMJ.

${ }^{1}$ Clinical Research Center \& Division of Mood Disorders, Shanghai Mental Health Center, Shanghai Jiao Tong University School of Medicine, Shanghai, China

${ }^{2}$ Shanghai Key Laboratory of Psychotic Disorders, Shanghai, China

${ }^{3}$ CAS Center for Excellence in Brain Science and Intelligence Technology, Shanghai, China

Correspondence to Dr Yiru Fang, Clinical Research Center \& Division of Mood Disorders, Shanghai Mental Health Center, Shanghai Jiao Tong University School of Medicine, CAS Center for Excellence in Brain Science and Intelligence Technology, Shanghai Key Laboratory of Psychotic Disorders, 600 South Wan Ping Road, Shanghai 200030, CHINA; yirufang@aliyun.com

\section{ABSTRACT}

Disturbance of biological rhythms contributes to the onset of bipolar disorders and is an important clinical feature of the condition. To further explore the role of biological rhythms in bipolar disorders, 95 English articles published between 1968 and 2019 were retrieved from the PubMed database and analysed. We herein review the outcomes of studies on biological rhythm disturbance in bipolar disorders, including the epidemiology, aetiology, clinical features (eg, sleep, feeding and eating disorders) and treatment of the condition evaluated by patients' selfreport and biological indicators such as melatonin. Our report supports the characterisation of biological rhythm disturbance as a significant clinical feature affecting the onset and development of bipolar disorders and reviews classical and novel treatments, such as chronotherapy, that can be applied in the clinical practice. Our analysis indicates that a more comprehensive study of the pathophysiology, clinical phenomenology and treatment of biological rhythm disturbance is required.

\section{INTRODUCTION}

Biological rhythms refer to cyclical variations in physiological and behavioural functions and include the daily sleep-wake cycle, hormonal metabolism, eating patterns and social activity, also known as circadian rhythm, and the ultradian and seasonal rhythm. Lifestyle-mediated disturbances of biological rhythms predispose individuals to several mental illnesses, including unipolar and bipolar disorders (BD). BD is a common, disabling mental illness with significant morbidity and mortality and an increased prevalence of suicide among the affected patients. The diagnostic criteria for $\mathrm{BD}$ are based on the patient exhibiting severe oscillations between high and low moods, which are referred to as (hypo)manic and depressive episodes, respectively. ${ }^{1} \mathrm{BD}$ can be categorised into BD type I, BD type II and subthreshold BD subtypes based on the duration and intensity of the episodes of high and low moods. According to the Diagnostic and Statistical Manual of Mental Disorders, Fourth Edition (DSM-IV), while cases of type I and type II BD share the same biological underpinnings, they are distinguished by the manifestation of an episode of mania or hypomania. ${ }^{12}$ The total lifetime (and 12-month) prevalence estimates across BD type I, BD typeII, and subthreshold BD subtypes have been reported to be $2.4 \%(1.5 \%){ }^{3}$ In a study including 67 373 individuals with BD from 44 countries conducted between 1980 and 2012, the lifetime prevalence for bipolar spectrum disorders was found to be $1.02 \%{ }^{4}$ The aetiology of $\mathrm{BD}$ is complex, and may involve an inherited predisposition, and/or social and psychological factors. ${ }^{2}$ Multiple studies suggest that the disruption of biological rhythms may also link to the onset, development, outcome and relapse of $\mathrm{BD} .^{5}$ Evidence indicates that BD could arise partially from a malfunction of the biological rhythm system, linking this disease with an abnormal 'clock' inside the body. ${ }^{5}$ The reciprocal relationship between the biological rhythm disturbance and $\mathrm{BD}$ is presented in figure 1.

The aim of this review is to identify research articles on the association between $\mathrm{BD}$ and biological rhythm disturbance indexed in the PubMed database from 1968 to 2019. The shortcomings of previous studies of BD are also discussed.

We adopted a broad scope for retrieving literature relevant to $\mathrm{BD}$ and biological rhythms. Our final collection included both human and animal research studies, reviews, meta-analyses and case reports. The PubMed database (https://www.ncbi.nlm.nih.gov/ pubmed) was used for retrieving relevant articles published until March 2019. The keywords used were as follows: 'bipolar disorders', 'sleep', 'feeding and eating disorders', 'melatonin', 'chronotherapy', 'cortisol', 'interpersonal and social rhythm therapy' and 'agomelatine'. In addition, the references of each article were manually checked for additional recent and relevant articles.

The literature search retrieved more than 1000 articles. After the exclusion of non-full 


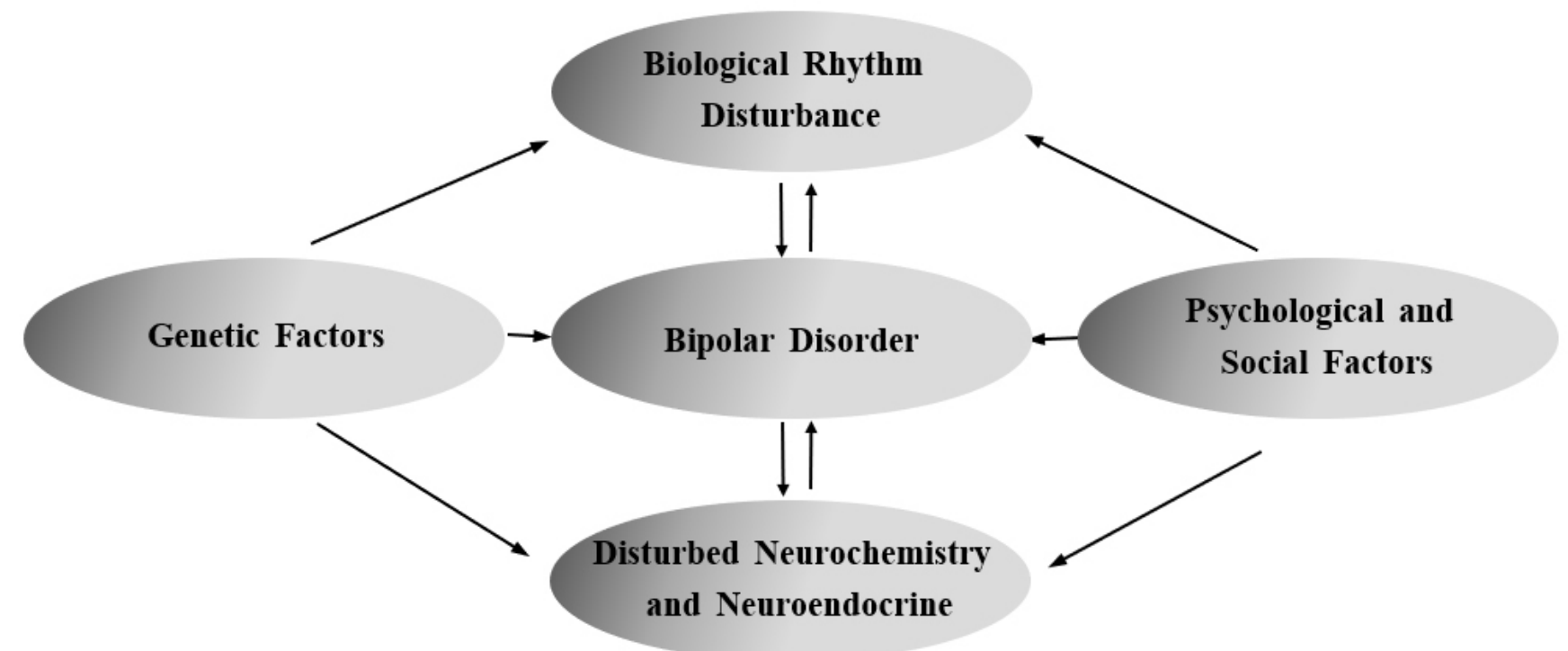

Figure 1 The reciprocal relationship between biological rhythm disturbance and bipolar disorders.

articles, abstracts were assessed for relevance. We thereby collected 95 studies that exploded the relationship between BD and biological rhythms for further analysis. The following paragraphs describe the clear role of biological rhythm dysfunction in the development and management of $\mathrm{BD}$.

\section{Influence of biological rhythm disturbance on BD}

Circadian misalignment might contribute to the pathophysiological mechanisms underlying the onset of $\mathrm{BD}$ as deduced by exploring the relationships among the disturbance of biological rhythms, cortisol levels and aberrant gene expression from saliva and buccal cell samples at four different time points for 2 consecutive days. ${ }^{6}$ The literature also suggested that the disruption of social factors (eg, interpersonal relationships, tasks, hobbies, habitation, life events and daily routines) might destabilise the circadian rhythms and aggravate mood episodes in patients with BD. ${ }^{6}$ One study reported that irregularity in social rhythms enhanced the vulnerability of at-risk adolescents to the onset of $\mathrm{BD}$ and that it may be possible to identify these at-risk adolescents before the first onset of $\mathrm{BD}$ based on their hypersensitivity to reward and irregularities in their social rhythms. ${ }^{6}$ A report of survival analyses indicated that the extent of irregularity in social rhythms significantly predicted the time to the first onset of hypomanic, manic and major depressive episodes. ${ }^{7}$

According to research, circadian rhythm sleep disorders feature complex multifactorial associations with affective disorders. Phenomenological investigations have observed variability in the duration and fragmentation of sleep as well as a high prevalence of shorter sleep times among high-risk individuals. ${ }^{8}$ Furthermore, sleep disturbance may be a prognostic indicator of the development of BD among high-risk youths (6-18 years old), ${ }^{9}$ and a phase delay in the rhythms of the melatonergic system has been associated with the onset of BD. Talih et al reported that dysfunctional melatonin and/or circadian biomarkers (eg, brain-derived neurotrophic factor) may also be involved in the pathophysiology of BD on account of their pivotal roles in delayed sleep phase syndrome. ${ }^{10}$

Biological rhythm disturbance persists across all stages of $\mathrm{BD}$, and over $80 \%$ of patients with $\mathrm{BD}$ reportedly exhibit dysfunction of biological rhythms. ${ }^{11}$ A systematic review found that individuals at high risk for BD often experience irregular circadian rhythms. ${ }^{12}$ Biological rhythm disruptions and sleep-associated difficulties have also been reported in patients with other mood and affective disorders like depressive disorder. ${ }^{13}$

A study examined the association between the disruptions in biological rhythms and the severity of depression by administering the Biological Rhythms Assessment in Neuropsychiatry to patients with depression. They observed increased biological rhythm disturbance, sleep disruption and disordered eating in patients with depression relative to healthy controls. Individuals with acute depression often displayed increased biological rhythm disturbance as compared with patients with depression in remission, and the least disturbance was reported in healthy controls. This observation suggests that disturbances in cyclical processes persist even during remission in patients with BD. ${ }^{14}$ The Systematic Treatment Enhancement Program for Bipolar Disorder study found that increased sleep variability was associated with more severe manic and depressive symptoms over a follow-up of 12 months and that daily activity and eating patterns normalise along with the remission of depression. ${ }^{14}$ Surprisingly, the disturbance of cyclical processes, such as loss of sleep, among individuals with a history of attempted suicide was predictive of higher level of suicidal ideation which is a risk factor of complete suicide. ${ }^{15}$

Disturbances in biological rhythms, such as loss of sleep, use of medication and severity of depression, diminish the quality of life among patients with BD. Furthermore, research on the neural correlates of wake-sleep regulation 
and cognition indicates that cognitive decay is directly related to negative symptomology in patients with $\mathrm{BD} .{ }^{16}$ This finding was supported by an additional study, which reported that disturbances in social rhythms may negatively affect the severity of depressive symptoms and may cause functional decay. ${ }^{14}$ With regard to the prognosis of patients with $\mathrm{BD}$, those with greater variability in their circadian cycle and daily and social activities exhibited worse psychosocial functioning. ${ }^{14}$ Thus, the influence of biological rhythm disturbance on the quality of life and prognosis of patients with $\mathrm{BD}$ warrants its consideration as a significant clinical feature. Treatment strategies aimed at normalising the biological rhythms were noted to improve the quality of life of patients with BD.

\section{Abnormalities in symptoms and clinical features associated with biological rhythm disruption \\ Circadian and seasonal variations in BD}

Patients with $\mathrm{BD}$ are vulnerable to disruptions in the sleep-wake cycle. They tend to engage in nocturnal activity and have irregular life schedules. It is well known that these disruptions in the sleep-wake cycle may aggravate the clinical symptoms of $\mathrm{BD}$ as it progresses. ${ }^{17}$

The Composite Scale of Morningness (CSM) is a common measure for evaluating circadian preference. ${ }^{17} \mathrm{~A}$ 2016 study revealed that compared with healthy controls, patients with BD had significantly lower CSM scores and showed a greater preference for evening activity and more irregular sleep-rise times. ${ }^{17}$ These features were similar across different age groups and between patients with $\mathrm{BD}$ I and BD II. ${ }^{18}$ In terms of the variability of sleep and wake times, patients with $\mathrm{BD}$ exhibited greater variability during weekdays/weekends compared with healthy controls. ${ }^{17}$ Evening preference was also associated with traits such as anxiety and poor social adjustment, which presented more often in patients with BD II than in those with BD I. ${ }^{17}$ A cohort study of twins with $\mathrm{BD}$ and co-twins without affective disorders found that the former had seasonally variable sleep length and higher global seasonality scores-a measure of the fluctuation in symptoms due to the season or time of the year-compared with the latter. ${ }^{19}$ One general population-based study has shown that evening preference was associated with depressive symptoms and higher proportions of body fat. ${ }^{20}$ These results indicate that patients with $\mathrm{BD}$ were prone to biological rhythm disruptions that resulted in negative symptomology.

Three studies have reported that seasonality was correlated with evening preference and abnormal circadian rhythms. ${ }^{172021}$ Lee et $a l^{22}$ reported that participants with suspended sleep phase syndrome demonstrated a greater likelihood of exhibiting variable symptom severity with seasonal variations. ${ }^{22}$ This might be explained by the phase shift hypothesis of seasonal affective disorder: depression in winter might be caused by an abnormally short exposure to natural light. ${ }^{23}$ Natale $e t a l^{21}$ suggested that individuals with evening preference and who had a persistently delayed circadian phase were more susceptible to seasonal affective disorder. ${ }^{21}$ In addition, personal and psychosocial factors may contribute to the regularity of the sleep-wake cycle and seasonality. The sleep-wake cycle may benefit from regular lifestyle and habits, whereas evening preference and aberrant bedrise time might aggravate seasonality by reducing the amount of light exposure. ${ }^{17}$ Providing further evidence for the seasonality of $\mathrm{BD}$, a study on 39 pairs of twins with $\mathrm{BD}$ found that $31 \%$ of them were hospitalised during autumn; however, no significant differences were found in the distribution of depressive and manic episodes according to admission. Hospitalisations due to depressive episodes were most frequent in autumn, while those due to manic episodes were common in autumn, spring and summer ( $86 \%$ of all episodes; 29 patients).${ }^{19}$ Hence, both circadian and seasonal rhythm-related disturbances influence BD and may contribute to the biopsychosocial basis of BD development.

\section{Sleep and $B D$}

Clinical and polysomnographic (PSG) studies of sleep in patients with BD have demonstrated that sleep disorder was a prominent clinical feature during acute depressive and manic episodes as well as through phases of remission. ${ }^{24}{ }^{25}$ PSG studies have demonstrated that normal sleep stages could be characterised by rapid eye movement (REM) and non-REM (NREM) sleep, which alternates in a cyclic pattern. This normal sleep cycle could be disrupted in both depressive and manic episodes of BD.

Actigraphy studies provided insight into the changes in the bedtime, sleep duration, sleep latency and waking after sleep onset in patients with $\mathrm{BD}$ (table 1). Nine clinical studies consistently concluded that patients with BD experienced poorer sleep efficiency than their healthy counterparts. ${ }^{25-33}$ Manic episodes were characterised by reduced sleep time. Patients undergoing manic episodes exhibited inferior sleep efficiency, longer sleeponset latency and reduced sleep time relative to normal controls but not to patients with depression, whose sleeping patterns were generally worse than those undergoing manic episodes. ${ }^{33}$ Although PSG findings were conflicting, ${ }^{34}$ individuals with BD have consistently been observed to exhibit greater abnormalities during manic episodes relative to their premorbid states, including a significantly decreased total sleep time, shortened REM latency and increased REM density. ${ }^{34}$ Abnormal sleep time may be a risk factor for the recurrence of manic or depressive episodes. A study of predictors of manic/hypomanic relapse in 159 adults found excessive sleepiness to be a predictor of mania, while hypersomnia demonstrated no such predictive value. ${ }^{35}$ Hence, these outcomes have significant implications for BD treatment counselling.

Retrospective studies of BD have consistently implicated hypersomnia, insomnia and early awakening as frequent symptoms, with rates of between $77 \%$ and $90 \%$ during depressive episodes. ${ }^{36}$ Sleep-related problems were reported in 49 of 80 patients (61.3\%) during their worst depressive episode. The sleep of individuals 


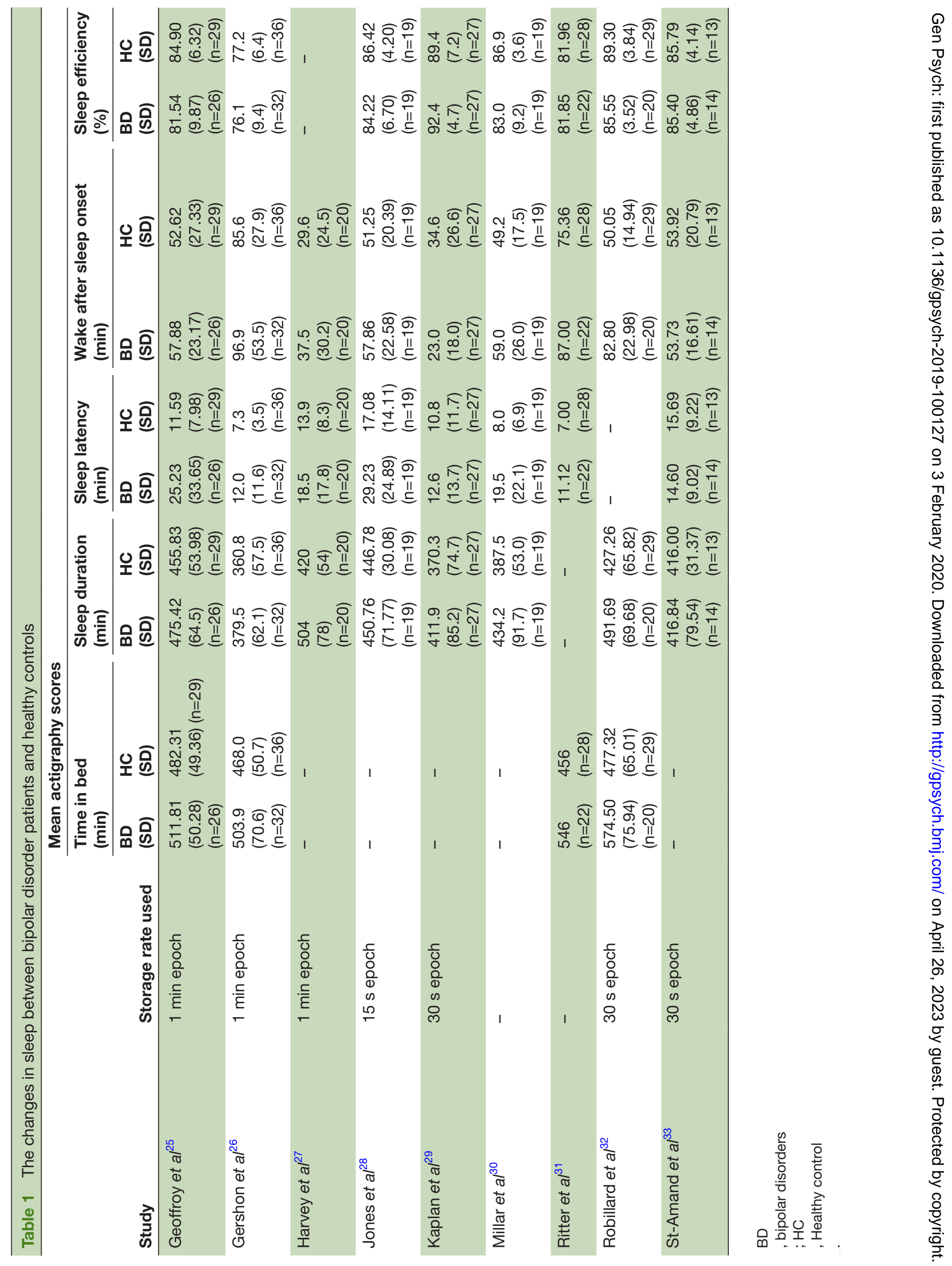


with BD was fragmented by REM disinhibition, reduced slow-wave sleep duration and impaired sleep continuity. REM disinhibition shortened the latency of REM sleep and prolonged REM duration. Impairments in the sleep continuity included extended sleep latency, early morning awakenings and an increased number of intermittent arousals. ${ }^{37}$ In addition, REM latency seemed to increase among patients with persistent suicidal ideation. Elevated suicidal ideation among patients with BD has been associated with a longer duration of NREM stage 1 sleep. ${ }^{38}$ Individuals with BD exhibited a greater degree of sleep disturbance at onset and throughout the course of their condition relative to individuals without $\mathrm{BD}{ }^{39}$ Regardless of their current mood state, patients with BD experienced more interrupted sleep, longer sleep-onset latency and less stability in bedtimes and awakening times as compared with healthy controls. These outcomes were evident in both individuals with a current mood state of any polarity and euthymic individuals with BD. Moreover, Merikangas et al reported that higher sad mood level was associated with longer sleep duration in those with BD type I but with shorter sleep duration in controls, based on examination of directional associations. ${ }^{40}$ The above findings suggested the importance of the management of sleep disturbance in patients with $\mathrm{BD} .^{39}$

\section{Eating patterns associated with $B D$}

The presence of comorbid eating disorders was common among individuals with BD. A study of the impact of $\mathrm{BD}$ on the severity of eating disorders identified binge eating disorders, bulimia nervosa and other eating disorders were not specified as variants of aberrant eating behaviour strongly associated with $\mathrm{BD} .{ }^{41}$ Another study found that $27 \%$ of patients with BD had a current DSM-V eating disorder, $12 \%$ had binge eating disorder, $15 \%$ had bulimia nervosa and $0.2 \%$ had anorexia nervosa. ${ }^{42}$ Moreover, night eating syndrome was reportedly more frequent among patients with BD than healthy controls. ${ }^{43}$ These conditions posed serious somatic problems related to being overweight or obese, such as diabetes or cardiovascular events. ${ }^{43}$ Binge eating behaviours among patients with BD were associated with a pattern of increased emotional instability, impulsivity and reactivity. ${ }^{44}$ In addition, a study of 175 patients found that obese patients with $\mathrm{BD}$ were more likely to have more severe and difficult-to-treat affective episodes as well as to experience the recurrence of $\mathrm{BD}$ with a predominance of depressive symptomology than were non-obese patients with BD. ${ }^{45}$ Meal timing was an important part of the body's circadian 'clock'. Moreover, aliment restriction influenced the clock genes in the peripheral tissues (peripheral 'clock'), but not those in the central nervous system (central 'clock'); thus, it was a strong indicator of possible desynchronisation between the peripheral and central clocks. This observation suggested that meal timing in the peripheral tissues might directly impact the coordination of the circadian rhythms. ${ }^{46}$
Patients with BD reportedly had greater difficulty in eating a healthy diet than those without $\mathrm{BD}$, and the consumption of tobacco, soft drinks or alcohol might be linked to the severity of $\mathrm{BD} .{ }^{47}$ Patients with $\mathrm{BD}$ I demonstrated less healthy eating habits than do those with BD II. $^{48}$ Hence, our review indicated that eating disorders were common among patients with $\mathrm{BD}$, unhealthy eating patterns were associated with poor emotional regulation and that the relationship between $\mathrm{BD}$ and eating disorders was bidirectional. Further research, especially prospective studies of factors such as impulsive eating that reportedly worsen outcomes in patients with $\mathrm{BD}$, was necessary to minimise the impact of eating disorders on the health of individuals with $\mathrm{BD}$.

\section{Social rhythms in $B D$}

Ehlers et al have defined social rhythms as social and interpersonal relationships, tasks or demands that entrained biological rhythms. ${ }^{49}$ Social rhythms affected the timing of biological clocks and thereby synchronised biological rhythms. A study involving healthy adults has shown that the regularity of daily life was associated with subjective sleep quality, but not with certain personality features such as neuroticism or extraversion. ${ }^{50}$

A 2014 study of more than 7000 German participants demonstrated that irregular social rhythms were significantly linked to greater anxiety, stress and depression. ${ }^{51}$ In addition, the disturbance of social rhythms may be implicated in the aetiology of BD. Consistent with the social zeitgeber (external forces) theory, the irregularity of social rhythms exacerbated the vulnerability of at-risk adolescents to the onset of BD. ${ }^{52}$ Similarly, another study demonstrated that the exposure to behavioural approach system (BAS) events predicted greater social rhythm disruption and higher levels of affective symptoms in individuals with high BAS sensitivity than those with moderate BAS sensitivity. ${ }^{53}$

As a study of mania has previously demonstrated, the affective phase was associated with more motor activity in novel situations. ${ }^{54}$ Research has further suggested that more severe manic symptoms correlated with increased irregularity in the rhythms of locomotor activity and lower frequency of daily activity, ${ }^{27}$ and that the disruptions in social rhythms were more frequent in patients undergoing a depressive phase relative to healthy individuals and those with euthymia. Bipolar depression has been associated with low activity levels, high variability of activity, retardation of circadian rhythms corresponding to both evening and morning activities and poor affective functioning. ${ }^{145}$ Similar to manic symptoms, the severity of depressive symptoms in patients with BD correlated with increased irregularity in social rhythms ${ }^{56}$ Furthermore, increased social rhythm disruptions were significantly associated with an increased risk of the recurrence of $\mathrm{BD} .{ }^{57}$ Although patients with $\mathrm{BD}$ often required medication to function normally, a more careful lifestyle assessment with attention to social rhythms and timing of activities might be critical for mood stability. ${ }^{58}$ 


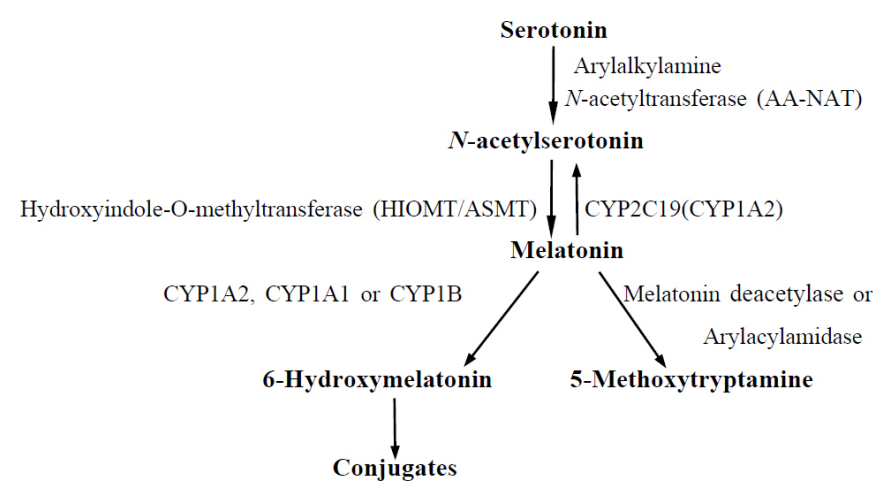

Figure 2 The in vivo metabolism of melatonin.

\section{Biomarkers and BD}

Biomarkers played a key role in the physiopathology of $\mathrm{BD}$ and major depressive disorder. Although two such biomarkers, melatonin and cortisol, have been considerably studied, and actual pharmacotherapeutics and psychotherapies targeting the normalisation of biomarkers have been developed over the past decades, the immunological role of such biomarkers in BD remained unclear.

\section{Melatonin}

It was hypothesised that abnormalities in circadian functioning and sleep were rooted in genetic factors that influenced the catecholamine loop circuit and caused affective disturbances. ${ }^{59}$ Melatonin, a neurohormone primarily synthesised by the pineal gland during the dark phase (at night), was the most direct measure of the state of the circadian system. ${ }^{59}$ Melatonin was synthesised by the conversion of serotonin to $\mathrm{N}$-acetylserotonin by arylalkylamine $N$-acetyltransferase and was subsequently converted to melatonin by acetyl serotonin O-methyltransferase (ASMT), also known as hydroxyindole O-methyltransferase ${ }^{60}$ Figure 2 illustrates the in vivo metabolism of melatonin.

Studies have shown that patients with BD exhibit diminished ASMT activity relative to healthy controls. Moreover, several deleterious ASMT mutations associated with low ASMT activity have been identified in patients with BD. Thus, ASMT could play a significant role in mediating vulnerability to $\mathrm{BD}$, and aberrations in the expression of melatonin may increase an individual's susceptibility to BD. ${ }^{60}$ However, this has not been investigated in humans. Melatonin contributed to the circadian clock by directly impacting the REM sleep atonia ${ }^{61}$ and then modulating the sleep-wake cycle. ${ }^{5}$ Its secretion was affected by dark environments; serum melatonin levels began to rise shortly after nightfall and peaked during the night (approximately between 02:00 and 04:00). Its secretion might decrease as a result of environmental light, disease, pineal calcification or age. ${ }^{61}$ Many studies have found changes in serum melatonin concentration in individuals with $\mathrm{BD}$; lower melatonin levels were significantly associated with all stages of $\mathrm{BD}$, including euthymia. ${ }^{62} \mathrm{~A}$ subsequent independent investigation reported that euthymic patients with BD had lower melatonin levels than did healthy controls when the study participants were awakened at 02:00 and required to sit in a cardiac chair from 02:00 to 04:00 ${ }^{61}$ Moreover, individuals with BD exhibited a later peak time of melatonin secretion at night than did healthy controls. ${ }^{62}$ The levels of melatonin at 15:00 and 19:00 hours in manic states were significantly higher than those in individuals with depression or controls, but were significantly lower at 07:00 hours. ${ }^{63}$ Additionally, the dim light melatonin onset was regarded as the most accurate marker for assessing the circadian pacemaker as the correlation was found between the magnitude of phase advances of dim light melatonin onset after morning light and the improvement in depression rating by an earlier report. ${ }^{64}$ Thus, we could conclude that melatonin was one of the biomarkers associated with BD and was a helpful tool to assess mood disorders.

Hence, our review found that certain differences were identified in the functional state of the melatonin system between patients with $\mathrm{BD}$ and healthy individuals, and these differences depended on whether the patient was undergoing a manic or depressive episode.

\section{Cortisol}

Cortisol was a glucocorticoid that acted on multiple organ systems to redirect energy resources to meet real or perceived threats. ${ }^{65}$ The negative feedback effects of the hypothalamo-pituitary-adrenocortical (HPA) axis and metabolism of cortisol were described in figure 3 . The dysfunction of the HPA axis has been identified as a physiological factor in the pathogenesis of $\mathrm{BD}$, including aberrations in the diurnal rhythm of cortisol and cortisol responses to stress or pharmacological manipulation. According to a biochemical study of the levels of urinary-free cortisol, cortisone and their metabolites, the enhanced clearance of cortisol was demonstrated to be a component of HPA axis hyperactivation as observed by increases in $5 \beta$-reductase, 11ßHSD 2 and $5 \alpha$-reductase levels. Significant between-group differences were found for cortisol metabolite indices, and the increased activity of $5 \beta$-reductase and 11ßHSD2 was noted, with a similar trend in the levels of $5 \alpha$-reductase in patients with BD compared with healthy controls. This dysfunction within the HPA axis was speculated to be a pathophysiological feature of $\mathrm{BD} .{ }^{66}$ Several studies have assessed the peripheral levels of cortisol, such as those in the blood serum, plasma or saliva. For example, a meta-analysis of 19 studies found that patients with non-manic BD exhibited small increases in morning cortisol levels relative to those of healthy controls. ${ }^{67}$ However, one study found no differences between the cortisol levels in the saliva or hair among patients with manic, depressive or euthymic $\mathrm{BD},{ }^{68}$ indicating that variances in cortisol levels were independent of mood state.

In cases of the recurrence of $\mathrm{BD}$, abnormalities in cortisol levels persisted after symptomatic recovery. This may be related to the number of previous $\mathrm{BD}$ episodes, as those with a greater number of previous episodes had 


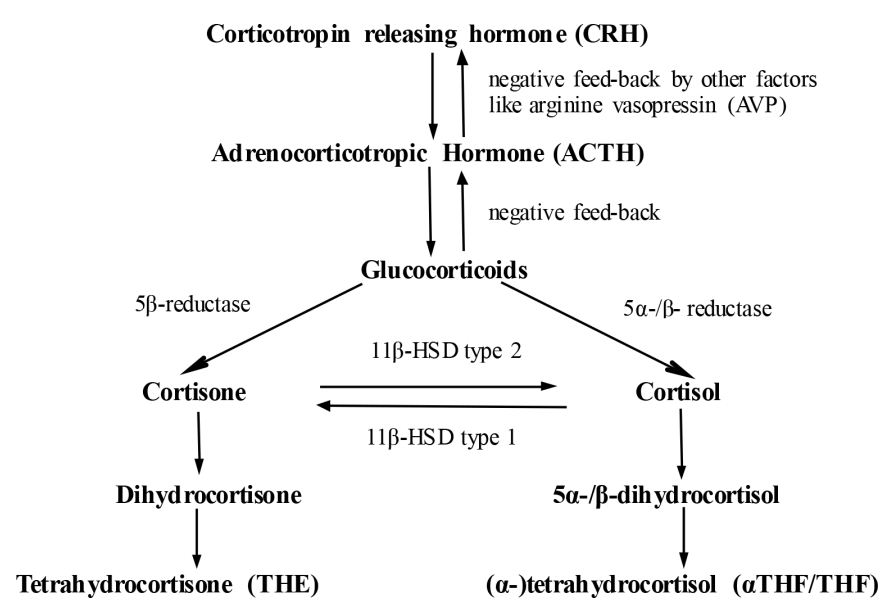

Figure 3 The negative feedback effects of the hypothalamopituitary-adrenocortical (HPA) axis and the metabolism of cortisol.

a higher mean level of cortisol, a blunted response to negative events and flatter cortisol diurnal slopes relative to patients with fewer episodes. ${ }^{69}$ Cortisol secretion in patients with $\mathrm{BD}$ showed more daily variation than did healthy controls. However, both the daytime cortisol levels and reactivity to daily events were similar between the remitted patients with $\mathrm{BD}$ and healthy controls. These results provided additional evidence of subtle HPA axis dysregulation in patients with remitted $\mathrm{BD}$, especially in those with many recurrent episodes. ${ }^{69}$

Cortisol awakening response (CAR), recognised as a reliable biological marker for examining the regulation of HPA axis feedback, was measured in individuals with BD after 45 min of waking. ${ }^{70}$ Patients with BD experiencing depressive symptoms had distinctly low levels of blunt CAR and cortisol levels relative to healthy controls. By contrast, the CAR, waking cortisol levels and immediate post-CAR decline did not differ between patients with $\mathrm{BD}$ and healthy controls.

The above observations indicated links among cortisol secretion patterns and different variables of $\mathrm{BD}$. These observations further provided evidence of HPA axis dysregulation in patients with acute $\mathrm{BD}$ and remittent patients with BD. However, many studies reported conflicting information. Hence, the mechanisms regulating the HPA axis warranted further research to identify possible targets for treating different stages of BD.

Overall, hormones are fundamental elements of the circadian system, and as abnormalities in hormonal profiles have been suggested to be characteristic of $\mathrm{BD}$, balancing these hormones may be key to treating the disease.

\section{Treatment of biological rhythm dysfunction}

Having a stable relationship between external (environmental variables capable of entraining circadian rhythms) and internal (activity and sleep patterns) factors is crucial to achieving a normal or euthymic mood state. Chronobiology researchers have highlighted the importance of timekeepers in maintaining this stability and have reported light and melatonin as the most significant contributors. Research on biological rhythms and sleep has led to the development of pharmacotherapies like agomelatine, and non-pharmacological therapies such as chronotherapy and interpersonal and social rhythm therapy (IPSRT), which can be used in everyday practice to treat affective disorders. ${ }^{62}$

\section{Chronotherapy}

Chronotherapy is based on controlled exposure to environmental stimuli that influences biological rhythms. This therapeutic method includes three basic techniques with proven efficacy, which are partial or total sleep deprivation (also referred to as wake therapy), sleep phase advance and light therapy. ${ }^{71}$ Sleep deprivation therapy directly targets the sleep-wake rhythm and influences the function of the suprachiasmatic nuclei by modifying vigilance state transitions and sleep states. It is clinically synergistic with, and may even act through the same mechanism as, morning light therapy, which identifies light as the main environmental synchroniser that affects the internal clock. The acute response rates to this therapy are similar to those reported for drug treatmentsthat is, from $2 / 3$ to $3 / 4$ among $\mathrm{BD}$ patients in depressive episode. ${ }^{71}$ However, unlike pharmacological treatments, which can take weeks to show efficacy, sleep deprivation therapy has a more rapid effect. ${ }^{71}$ Sit et al ${ }^{72}$ reported that the group treated with a bright white light experienced a significantly higher remission rate at 4-6 weeks than the placebo light group. ${ }^{72}$ An investigation conducted over a 7-week period indicated the combined administration of sleep deprivation, bright light and sleep phase advanced therapies significantly attenuated depressive symptoms relative to a group that only received medication within 48 hours of sleep deprivation. ${ }^{73}$

According to the phase advance hypothesis of depression, a state of earlier and more synchronised rhythmic variables disturbs the usual circadian rhythm, which subsequently may be unable to entrain to the 24 hours' external day-night cycle. During an episode of affective disorder, there is an asynchrony of the remaining sleepwake cycle in a normal or phase-delayed position, which tends to advance to the sleep phase. ${ }^{73}$

Clinical observations and research suggested that daily doses of light exposure could rapidly and profoundly alleviate mood disorders and sleep irregularities. ${ }^{73}$ Clinicians recommended initiating treatment with $15 \mathrm{~min}$ of midday light to attenuate the severity of $\mathrm{BD} .{ }^{73}$ Contraindications for light therapy were rare, and its compatibility with medication and other psychiatric treatments suggested that it could be integrated into standard practice. One study has attested the curative effect of bright light therapy (BLT) and dim light therapy. According to the criteria for remission and a decrease in the scores of the Hamilton Depression Rating Scale and MontgomeryAsberg Depression Rating Scale, response rates were 81\% and $19 \%$ while remission rates were $44 \%$ and $12.5 \%$, respectively. The results indicated that BLT was a safe 
and effective adjunctive treatment for $\mathrm{BD}$ with a seasonal pattern. ${ }^{74}$ Another study found that adolescents with BD with breakthrough depressive symptoms might benefit from light therapy when administered in conjunction with continued antidepressant treatment. ${ }^{75}$ However, one study provided robust evidence that confirmed the efficacy of midday BLT in treating BD but demonstrated that women with $\mathrm{BD}$ were highly sensitive to morning BLT. ${ }^{76}$

\section{Interpersonal and social rhythm therapy}

A comprehensive understanding of rhythm disturbances consequent of BD may lead to the development of chronobiologically based interventions to treat this disorder. ${ }^{77}$ As posited by the social zeitgeber model of $\mathrm{BD}$, social rhythms entrained circadian rhythms, the stabilisation of which is thought to improve affective symptoms and episodes. This theory established the foundation of IPSRT, which was developed by Frank $e t a l^{78}$ to help patients with $\mathrm{BD}$ address interpersonal problems and regulate their social rhythms by restabilising daily routines and normalising the sleep-wake cycle. ${ }^{78}$ This was a disorder-specific form of psychotherapy based on the instability model of BD. It addressed three mechanisms of relapse: disrupted social rhythms, non-adherence to the medication regime and interpersonal life events.

Although prior research suggested that this method might be particularly beneficial for adolescents at high risk for $\mathrm{BD},{ }^{79}$ another study on the willingness of adolescents with a family history of BD reported that $67 \%$ of families declined therapy based on the adolescent's belief that he/she did not need therapy, indicating that IPSRT might not be an acceptable prophylactic option among adolescents. ${ }^{80}$ However, the investigation did not obtain clear reasons for adolescents having declined. Some clinical trials aiming to estimate the efficacy of IPSRT as an adjunctive treatment found that it could ameliorate manic and depressive symptoms and improve social functioning in young adults and adolescents with $\mathrm{BD}$ and high rates of comorbidity. ${ }^{81}$ Individuals who received IPSRT in the acute phase of treatment survived longer without a new episode of mood disorder. IPSRT might contribute favourably to the clinical management of $\mathrm{BD}$, particularly with the prevention of recurrence. Reviewing the courses of patients' illnesses and interpersonal problems, Hoberg et al used the data to inform structured activity that could improve the patients' interpersonal relationships and stabilise their daily routines. ${ }^{82}$ After a 12-week follow-up assessment, depressive symptoms and psychosocial functioning had improved considerably, and the participants reported high satisfaction with IPSRT. However, while mania or illness severity of BD showed a trend towards improvement from baseline to 12 weeks, only $16 \%$ of the sample met the criteria for the remission of symptoms of depression. ${ }^{82}$ Another IPSRT study was conducted over a longer duration with 2-hour weekly sessions for 1 year; the researchers found that the participants who underwent the sessions experienced significant decreases in depressive symptoms and exhibited normalisation of social rhythms during the treatment duration..$^{82}$ However, no significant improvement in manic symptoms was observed. These three clinical studies suggested that IPSRT might be effective for the attenuation of depressive symptoms. Additional randomised controlled trials with a special control group, such as those who underwent other psychosocial interventions, were necessary to ascertain the efficacy of IPSRT.

IPSRT is recommended as a useful tool for stabilising episodic fluctuations and delaying the recurrence of affective symptoms in patients with $\mathrm{BD}$. With its emphasis on increasing the regularity of social routines and thereby possibly enhancing circadian integrity, IPSRT provides a feasible approach to $\mathrm{BD}$ treatment by systematically enhancing the regularity of all the patient's daily routines. ${ }^{83}$

\section{Agomelatine}

First reported in 1992 among a series of synthetic naphthalene melatonin analogues, agomelatine ( $N$-[2-(7methoxynaphth-1-yl) ethyl] acetamide) acted as a potent agonist for melatonin (MT1 and MT2) and serotonin (5-hydroxytryptamine and 5-hydroxytryptamine2C) receptors. ${ }^{84}$ Specifically, agomelatine mediated the chronobiological effect via melatonin receptors in the suprachiasmatic nucleus of the hypothalamus, known as the 'endogenous biological clock'. ${ }^{84}$ Experiments demonstrate the drug's capacity to reset disorganised circadian rhythms in several behavioural animal models. ${ }^{85} \mathrm{~A}$ previous study showed that 10 years after the initiation of melatonin treatment for chronic sleep-onset insomnia, approximately $75 \%$ of the patients gained normal sleep quality without medication. ${ }^{86}$ Another study emphasised that the application of melatonin therapy to help treat shift employees who experienced difficulty in falling asleep could significantly improve sleep-onset latency and sleep efficiency. ${ }^{87}$ These findings indicated that agomelatine might be used as an adjunctive treatment for BD and possibly prevent its recurrence when administered during remission phases, especially among those who valued selfmanagement and independence. In addition, agomelatine might help prevent metabolic syndromes in patients treated with antipsychotics. ${ }^{24} 88$ Only low doses of melatonin have been evaluated, and doses of 2-6 $\mathrm{mg}$ were generally necessary to yield a clinical effect. One study reported that a dose of 1-10 mg of melatonin could increase serum melatonin concentrations by 10-100 times within 1 hour of administration, and its concentrations sloped to baseline at roughly $4-8$ hours after administration. ${ }^{61}$ These findings suggested that the modulation of melatonin receptors might provide an effective, targeted method of treating BD. Some clinical studies have confirmed that agomelatine was effective for treating both BD I and BD II. Twenty-five milligrams/day of agomelatine was well tolerated when supplemented with valproic acid/lithium treatment to treat acute depression in patients with BD I or BD II. ${ }^{89}$ Although agomelatine showed efficacy similar to that of classic antidepressants and an acceptable safety, ${ }^{90}$ a subsequent induction of mania was reported 
in one case. Kennel $e t a \ell^{11}$ surmised that this was caused by the interaction between ethynyloestradiol, which was a component of the contraception used by this quadragenarian patient, and the cytochrome P450 1A2 substrate of agomelatine. ${ }^{91}$ Other risks associated with agomelatine, such as headache, diarrhoea, insomnia, anxiety, hepatotoxicity and the need to monitor liver function during the administration period, should be noted. ${ }^{92}$

\section{Other pharmacotherapies}

Multiple effective pharmacotherapies exist for the treatment of BD. Mood stabilisers, including lithium, antiepileptics such as valproic acid, antipsychotics like quetiapine, hypnotics and anxiolytics, are often used depending on the symptoms of BD exhibited by the patient. Some mood stabilisers and antipsychotics affect several circadian or biological parameters, including peak activity time and robustness of circadian rhythms.

For example, lithium is still considered the most effective drug for managing both bipolar mania and depression. It has been recommended as a first-line treatment by the Canadian Network for Mood and Anxiety Treatments and the International Society for Bipolar Disorders, which has suggested that serum lithium levels should be maintained between 0.8 and $1.2 \mathrm{meq} / \mathrm{L} .{ }^{3}$ A genetic study further confirmed the relationship between lithium and circadian rhythms. The expression of Per2, Cry 1 and Reverba, which are constituents of the circadian clock, was influenced by lithium treatment. ${ }^{93}$ A systematic review has shown that chronic lithium treatment might stabilise the rhythms of circadian activity by delaying circadian rhythms and reducing the peak body temperature, as well as reducing the amplitude and duration of activity rhythms. ${ }^{94}$ Previously, with a proven level I evidence of its efficacy, quetiapine was recommended as the firstline treatment, and 2018 guidelines advised clinicians to consider a target dose of $300 \mathrm{mg} /$ day when treating patients with BD. ${ }^{3}$ Quetiapine addressed a broad spectrum of indications, including manic and acute psychosis. In clinical studies, quetiapine reportedly improved the quality of sleep. To verify its therapeutic action on circadian rhythmicity, 20 healthy volunteers received $150 \mathrm{mg}$ of quetiapine extended release for seven nights. The actigraphy of the volunteers revealed that quetiapine increased sleep duration and efficiency, prolonged final wake time and reduced within-day emotional variability in these volunteers relative to 20 matched controls who received a placebo. ${ }^{95}$

However, despite the multiple approaches adopted to treat $\mathrm{BD}$, addressing disruptions of biological rhythms in patients with $\mathrm{BD}$ remains a challenge. The controlled and integrated administration of stimuli that can directly influence the biological 'clock', such as light, melatonin, IPSRT and manipulations of the sleep-wake rhythm, has established high efficacy in the attenuation of mental episodes of both unipolar and bipolar disorders. This demonstrates that the relationship between the internal clock and mental episodes is of major relevance to the clinical treatment of $\mathrm{BD}$ and offers a new perspective on the management and treatment of the disorder in common clinical practice.

\section{FUTURE DIRECTIONS}

Although extensive research has been conducted on biological rhythm disturbance associated with $\mathrm{BD}$, multiple avenues of research remain to be explored. For example, the biological roots of circadian rhythm disruption remain controversial. Both exogenous (eg, environmental factors) and endogenous factors (eg, motor, energy, sleep) must be accounted for in order to explore the exact nature of this disturbance.

Moreover, some articles included in this review had certain limitations, such as small sample size, short observation periods, lack of all aspects of mood status and the absences of control groups. For example, few studies involved patients with a manic state. The paucity of evidence related to mania was possibly associated with the obvious clinical limitations of studying these patients. Most research on BD focuses on bipolar depression, which provides an overly narrow, biased foundation from which it is difficult to form an accurate theory of biological rhythm disturbance. In addition, lack of evaluation of the potential effects impacting the precise assessment of experimental variables in earlier studies may have contributed to the contradictory opinions in the literature. These studies did not adequately characterise BD or develop novel therapies for its treatment. In addition, many of these studies used discrepant methods and focused on different physiological and therapeutic parameters. These variances may further account for the difficulty in comparing their findings.

Theoretical approaches to the understanding of the biological rhythms also require further improvement. For instance, devising a concept of broader biological rhythms, inclusive of the 24 hours' circadian timeline and incorporating the ultradian (periods of $<24$ hours) and infradian (periods $>24$ hours) rhythms, ${ }^{77}$ requires further research and complete characterisation of rhythm disruptions in BD. Studies should collect data more frequently and over longer durations to fully characterise the biological rhythm disturbances in BD. Because of the limitations of current studies that we screened, most of the present review focused on the 'circadian rhythm' and only a small part discussed the seasonal variations in BD. Certainly, the recurrent nature of bipolar cycles, sometimes on an annual basis, suggests that some alteration of biological rhythms may under another cyclicity.

Whereas some clinical and animal research pointed to a disturbance of biological timing system in $\mathrm{BD}$, the truth that rhythm disturbance was not the only consequence existed. In fact, the biological rhythm system, especially the circadian timing system, was intertwined with both physiological system's in vivo and external environmental factors. Probably other physiological or environmental factors directly affected the destabilising biological 
rhythm, but not being the main disturbance in the biological timing system itself. This will be another challenge for future research in physiological domain that the association between other physiological processes and biological timing system should be noticed.

Although both preclinical and clinical studies have helped characterise the phenomenon of biological system dysfunction, as well as contribute to its resolution, the rate of recurrence of $\mathrm{BD}$ remained high. Hence, improving the lifetime cure rate must be prioritised in future studies.

\section{CONCLUSIONS}

Individuals with BD exhibit more biological rhythm disruptions at disease onset and across follow-up, relative to healthy controls. These disturbances were partly independent of BD subtype but were more evident in $\mathrm{BD}$ individuals with current affective disorder episodes. Biological and circadian disturbances are associated with psychosocial difficulties and can increase the risk of further affective episodes, thus warranting their early identification and management. Despite the availability of several pharmacotherapies and non-pharmacological treatments with demonstrable efficacy, novel and sound treatments are still required.

Acknowledgements We are deeply grateful to all participants and investigators included in this review's studies.

Competing interests None declared.

Patient consent for publication Not required.

Provenance and peer review Not commissioned; externally peer reviewed.

Open access This is an open access article distributed in accordance with the Creative Commons Attribution Non Commercial (CC BY-NC 4.0) license, which permits others to distribute, remix, adapt, build upon this work non-commercially, and license their derivative works on different terms, provided the original work is properly cited, appropriate credit is given, any changes made indicated, and the use is non-commercial. See: http://creativecommons.org/licenses/by-nc/4.0/.

\section{REFERENCES}

1 Baek JH, Park DY, Choi J, et al. Differences between bipolar I and bipolar II disorders in clinical features, comorbidity, and family history. J Affect Disord 2011;131:59-67.

2 Foley SF, Bracher-Smith M, Tansey KE, et al. Fractional anisotropy of the uncinate fasciculus and cingulum in bipolar disorder type I, type II, unaffected siblings and healthy controls. Br J Psychiatry 2018;213:548-54.

3 Yatham LN, Kennedy SH, Parikh SV, et al. Canadian network for mood and anxiety treatments (CANMAT) and International Society for bipolar disorders (ISBD) 2018 guidelines for the management of patients with bipolar disorder. Bipolar Disord 2018;20:97-170.

4 Moreira ALR, Van Meter A, Genzlinger J, et al. Review and metaanalysis of epidemiologic studies of adult bipolar disorder. J Clin Psychiatry 2017;78:e1259-69.

5 Harvey AG. Sleep and circadian rhythms in bipolar disorder: seeking synchrony, harmony, and regulation. Am J Psychiatry 2008;165:820-9.

6 Moon J-H, Cho C-H, Son GH, et al. Advanced circadian phase in mania and delayed circadian phase in mixed mania and depression returned to normal after treatment of bipolar disorder. EBioMedicine 2016;11:285-95.

7 Shen GHC, Alloy LB, Abramson LY, et al. Social rhythm regularity and the onset of affective episodes in bipolar spectrum individuals. Bipolar Disord 2008;10:520-9.
8 Ankers D, Jones SH. Objective assessment of circadian activity and sleep patterns in individuals at behavioural risk of hypomania. J Clin Psychol 2009;65:1071-86.

9 Levenson JC, Axelson DA, Merranko J, et al. Differences in sleep disturbances among offspring of parents with and without bipolar disorder: association with conversion to bipolar disorder. Bipolar Disord 2015;17:836-48.

10 Talih F, Gebara NY, Andary FS, et al. Delayed sleep phase syndrome and bipolar disorder: pathogenesis and available common biomarkers. Sleep Med Rev 2018;41:133-40.

11 Giglio LM, Magalhães PVS, Kapczinski NS, et al. Functional impact of biological rhythm disturbance in bipolar disorder. J Psychiatr Res 2010;44:220-3.

12 Melo MCA, Garcia RF, Linhares Neto VB, et al. Sleep and circadian alterations in people at risk for bipolar disorder: a systematic review. J Psychiatr Res 2016;83:211-9.

13 Melo MCA, Abreu RLC, Linhares Neto VB, et al. Chronotype and circadian rhythm in bipolar disorder: a systematic review. Sleep Med Rev 2017;34:46-58.

14 Pinho M, Sehmbi M, Cudney LE, et al. The association between biological rhythms, depression, and functioning in bipolar disorder: a large multi-center study. Acta Psychiatr Scand 2016;133:102-8.

15 Stange JP, Kleiman EM, Sylvia LG, et al. Specific mood symptoms confer risk for subsequent suicidal ideation in bipolar disorder with and without suicide attempt history: MULTI-WAVE data from STEPBD. Depress Anxiety 2016;33:464-72.

16 Martinez-Aran A, Vieta E, Torrent C, et al. Functional outcome in bipolar disorder: the role of clinical and cognitive factors. Bipolar Disord 2007;9:103-13.

17 Baek JH, Kim JS, Kim MJ, et al. Lifetime characteristics of EveningPreference and irregular Bed-Rise time are associated with lifetime seasonal variation of mood and behavior: comparison between individuals with bipolar disorder and healthy controls. Behav Sleep Med 2016;14:155-68.

18 Kim K, Weissman A, Puzia M, et al. Circadian phase preference in pediatric bipolar disorder. J Clin Med 2014;3:255-66.

19 Hakkarainen R, Johansson C, Kieseppä T, et al. Seasonal changes, sleep length and circadian preference among twins with bipolar disorder. BMC Psychiatry 2003;3:6.

20 Murray G, Allen NB, Trinder J. Seasonality and circadian phase delay: prospective evidence that winter lowering of mood is associated with a shift towards Eveningness. J Affect Disord 2003;76:15-22.

21 Natale V, Adan A, Scapellato P. Are seasonality of mood and eveningness closely associated? Psychiatry Res 2005;136:51-60.

22 Lee H-J, Rex KM, Nievergelt CM, et al. Delayed sleep phase syndrome is related to seasonal affective disorder. J Affect Disord 2011;133:573-9.

23 Lewy AJ, Sack RL, Singer CM, et al. Winter depression and the phase-shift hypothesis for bright light's therapeutic effects: history, theory, and experimental evidence. J Biol Rhythms 1988;3:121-34.

24 Geoffroy P, Etain B, Franchi J-A, et al. Melatonin and melatonin agonists as adjunctive treatments in bipolar disorders. Curr Pharm Des 2015;21:3352-8.

25 Geoffroy PA, Boudebesse C, Bellivier F, et al. Sleep in remitted bipolar disorder: a naturalistic case-control study using actigraphy. $J$ Affect Disord 2014;158:1-7.

26 Gershon A, Thompson WK, Eidelman P, et al. Restless pillow, ruffled mind: sleep and affect coupling in interepisode bipolar disorder. $J$ Abnorm Psychol 2012;121:863-73.

27 Harvey AG, Schmidt DA, Scarnà A, et al. Sleep-related functioning in euthymic patients with bipolar disorder, patients with insomnia, and subjects without sleep problems. Am J Psychiatry 2005;162:50-7.

28 Jones SH, Hare DJ, Evershed K. Actigraphic assessment of circadian activity and sleep patterns in bipolar disorder. Bipolar Disord 2005;7:176-86.

29 Kaplan KA, Talbot LS, Gruber J, et al. Evaluating sleep in bipolar disorder: comparison between actigraphy, polysomnography, and sleep diary. Bipolar Disord 2012;14:870-9.

30 Millar A, Espie CA, Scott J. The sleep of remitted bipolar outpatients: a controlled naturalistic study using actigraphy. J Affect Disord 2004;80:145-53.

31 Ritter PS, Marx C, Lewtschenko N, et al. The characteristics of sleep in patients with manifest bipolar disorder, subjects at high risk of developing the disease and healthy controls. J Neural Transm 2012;119:1173-84.

32 Robillard R, Naismith SL, Rogers NL, et al. Delayed sleep phase in young people with unipolar or bipolar affective disorders. J Affect Disord 2013;145:260-3.

33 St-Amand J, Provencher MD, Bélanger L, et al. Sleep disturbances in bipolar disorder during remission. J Affect Disord 2013;146:112-9. 
34 Benca RM, Obermeyer WH, Thisted RA, et al. Sleep and psychiatric disorders. A meta-analysis. Arch Gen Psychiatry 1992;49:651-68.

35 Kaplan KA, McGlinchey EL, Soehner A, et al. Hypersomnia subtypes, sleep and relapse in bipolar disorder. Psychol Med 2015;45:1751-63.

36 Rocha PMB, Neves FS, Corrêa H. Significant sleep disturbances in euthymic bipolar patients. Compr Psychiatry 2013;54:1003-8.

37 Baskaran A, Summers D, Willing SL, et al. Sleep architecture in ziprasidone-treated bipolar depression: a pilot study. Ther $A d v$ Psychopharmacol 2013;3:139-49.

38 Bernert RA, Luckenbaugh DA, Duncan WC, et al. Sleep architecture parameters as a putative biomarker of suicidal ideation in treatmentresistant depression. J Affect Disord 2017;208:309-15.

39 Seleem MA, Merranko JA, Goldstein TR, et al. The longitudinal course of sleep timing and circadian preferences in adults with bipolar disorder. Bipolar Disord 2015;17:392-402.

40 Merikangas KR, Swendsen J, Hickie IB, et al. Real-time mobile monitoring of the dynamic associations among motor activity, energy, mood, and sleep in adults with bipolar disorder. JAMA Psychiatry 2019;76:190-8.

41 Thiebaut S, Jaussent I, Maimoun L, et al. Impact of bipolar disorder on eating disorders severity in real-life settings. J Affect Disord 2019;246:867-72.

42 McElroy SL, Crow S, Blom TJ, et al. Prevalence and correlates of DSM-5 eating disorders in patients with bipolar disorder. $J$ Affect Disord 2016;191:216-21.

43 Melo MCA, de Oliveira Ribeiro M, de Araújo CFC, et al. Night eating in bipolar disorder. Sleep Med 2018;48:49-52.

44 Boulanger H, Tebeka S, Girod C, et al. Binge eating behaviours in bipolar disorders. J Affect Disord 2018;225:482-8.

45 Fagiolini A, Kupfer DJ, Houck PR, et al. Obesity as a correlate of outcome in patients with bipolar I disorder. Am J Psychiatry 2003;160:112-7.

46 Abreu T, Bragança M. The bipolarity of light and dark: a review on bipolar disorder and circadian cycles. J Affect Disord 2015;185:219-29.

47 Huang J, Yuan CM, Xu XR, et al. The relationship between lifestyle factors and clinical symptoms of bipolar disorder patients in a Chinese population. Psychiatry Res 2018;266:97-102.

48 Bernstein EE, Nierenberg AA, Deckersbach T, et al. Eating behavior and obesity in bipolar disorder. Aust N Z J Psychiatry 2015;49:566-72.

49 Ehlers CL, Frank E, Kupfer DJ. Social zeitgebers and biological rhythms. A unified approach to understanding the etiology of depression. Arch Gen Psychiatry 1988;45:948-52.

50 Monk TH, Petrie SR, Hayes AMYJ, et al. Regularity of daily life in relation to personality, age, gender, sleep quality and circadian rhythms. J Sleep Res 1994;3:196-205.

51 Velten J, Lavallee KL, Scholten S, et al. Lifestyle choices and mental health: a representative population survey. BMC Psychol 2014;2.

52 Alloy LB, Boland EM, Ng TH, et al. Low social rhythm regularity predicts first onset of bipolar spectrum disorders among atrisk individuals with reward hypersensitivity. J Abnorm Psychol 2015;124:944-52.

53 Boland EM, Stange JP, Labelle DR, et al. Affective disruption from social rhythm and behavioral approach system (BAS) sensitivities: a test of the integration of the social Zeitgeber and BAS theories of bipolar disorder. Clin Psychol Sci 2016;4:418-32.

54 Minassian A, Henry BL, Geyer MA, et al. The quantitative assessment of motor activity in mania and schizophrenia. $J$ Affect Disord 2010;120:200-6.

55 Ashman SB, Monk TH, Kupfer DJ, et al. Relationship between social rhythms and mood in patients with rapid cycling bipolar disorder. Psychiatry Res 1999;86:1-8.

56 Grierson AB, Hickie IB, Naismith SL, et al. Circadian rhythmicity in emerging mood disorders: state or trait marker? Int J Bipolar Disord 2016;4.

57 Levenson JC, Wallace ML, Anderson BP, et al. Social rhythm disrupting events increase the risk of recurrence among individuals with bipolar disorder. Bipolar Disord 2015;17:869-79.

58 Soreca I, Wallace ML, Hall MH, et al. The association between meal timing and frequency with cardiometabolic profile in patients with bipolar disorder. Acta Psychiatr Scand 2016;133:453-8.

59 Pacchierotti C, lapichino S, Bossini L, et al. Melatonin in psychiatric disorders: a review on the melatonin involvement in psychiatry. Front Neuroendocrinol 2001;22:18-32.

60 Etain B, Dumaine A, Bellivier F, et al. Genetic and functional abnormalities of the melatonin biosynthesis pathway in patients with bipolar disorder. Hum Mol Genet 2012;21:4030-7.

61 McGrane IR, Leung JG, St Louis EK, et al. Melatonin therapy for REM sleep behavior disorder: a critical review of evidence. Sleep Med 2015;16:19-26.
62 Nurnberger Jl, Adkins S, Lahiri DK, et al. Melatonin suppression by light in euthymic bipolar and unipolar patients. Arch Gen Psychiatry 2000;57:572-9. Jr.

63 Nováková M, Praško J, Látalová K, et al. The circadian system of patients with bipolar disorder differs in episodes of mania and depression. Bipolar Disord 2015;17:303-14.

64 Pandi-Perumal SR, Smits M, Spence W, et al. Dim light melatonin onset (DImo): a tool for the analysis of circadian phase in human sleep and chronobiological disorders. Prog Neuropsychopharmacol Biol Psychiatry 2007:31:1-11.

65 Herman JP, McKlveen JM, Ghosal S, et al. Regulation of the hypothalamic-pituitary-adrenocortical stress response. Compr Physiol 2016;6:603-21.

66 Steen NE, Methlie P, Lorentzen S, et al. Altered systemic cortisol metabolism in bipolar disorder and schizophrenia spectrum disorders. J Psychiatr Res 2014;52:57-62.

67 Girshkin L, Matheson SL, Shepherd AM, et al. Morning cortisol levels in schizophrenia and bipolar disorder: a meta-analysis. Psychoneuroendocrinology 2014;49:187-206.

68 Manenschijn L, Spijker AT, Koper JW, et al. Long-Term cortisol in bipolar disorder: associations with age of onset and psychiatric comorbidity. Psychoneuroendocrinology 2012;37:1960-8.

69 Havermans R, Nicolson NA, Berkhof J, et al. Patterns of salivary cortisol secretion and responses to daily events in patients with remitted bipolar disorder. Psychoneuroendocrinology 2011;36:258-65.

70 Huang M-C, Chuang S-C, Tseng M-CM, et al. Cortisol awakening response in patients with bipolar disorder during acute episodes and partial remission: a pilot study. Psychiatry Res 2017;258:594-7.

71 Dallaspezia S, Benedetti F, Melatonin BF. Melatonin, circadian rhythms, and the clock genes in bipolar disorder. Curr Psychiatry Rep 2009:11:488-93.

72 Sit DK, McGowan J, Wiltrout C, et al. Adjunctive bright light therapy for bipolar depression: a randomized double-blind placebocontrolled trial. AJP 2018;175:131-9.

73 Wu JC, Kelsoe JR, Schachat C, et al. Rapid and sustained antidepressant response with sleep deprivation and chronotherapy in bipolar disorder. Biol Psychiatry 2009;66:298-301.

74 Yorguner Kupeli N, Bulut NS, Carkaxhiu Bulut G, et al. Efficacy of bright light therapy in bipolar depression. Psychiatry Res 2018;260:432-8.

75 Papatheodorou G, Kutcher S. The effect of adjunctive light therapy on ameliorating breakthrough depressive symptoms in adolescentonset bipolar disorder. J Psychiatry Neurosci 1995;20:226-32.

76 Zhou T-H, Dang W-M, Ma Y-T, et al. Clinical efficacy, onset time and safety of bright light therapy in acute bipolar depression as an adjunctive therapy: a randomized controlled trial. J Affect Disord 2018:227:90-6.

77 Gonzalez R. The relationship between bipolar disorder and biological rhythms. J Clin Psychiatry 2014;75:e323-31.

78 Frank E, Swartz HA, Boland E. Interpersonal and social rhythm therapy: an intervention addressing rhythm dysregulation in bipolar disorder. Dialogues Clin Neurosci 2007:9:325-32.

79 Goldstein TR, Fersch-Podrat R, Axelson DA, et al. Early intervention for adolescents at high risk for the development of bipolar disorder: pilot study of interpersonal and social rhythm therapy (IPSRT). Psychotherapy 2014;51:180-9.

80 Haynes PL, Gengler D, Kelly M. Social rhythm therapies for mood disorders: an update. Curr Psychiatry Rep 2016;18:75.

81 Inder ML, Crowe MT, Luty SE, et al. Randomized, controlled trial of interpersonal and social rhythm therapy for young people with bipolar disorder. Bipolar Disord 2015;17:128-38.

82 Hoberg AA, Ponto J, Nelson PJ, et al. Group interpersonal and social rhythm therapy for bipolar depression. Perspect Psychiatr Care 2013;49:226-34

83 Hlastala SA, Frank E. Adapting interpersonal and social rhythm therapy to the developmental needs of adolescents with bipolar disorder. Dev Psychopathol 2006;18:1267-88.

84 Redman JR, Francis AJ. Entrainment of rat circadian rhythms by the melatonin agonist S-20098 requires intact suprachiasmatic nuclei but not the pineal. J Biol Rhythms 1998;13:39-51.

85 Tchekalarova J, Stoynova T, llieva K, et al. Agomelatine treatment corrects symptoms of depression and anxiety by restoring the disrupted melatonin circadian rhythms of rats exposed to chronic constant light. Pharmacol Biochem Behav 2018;171:1-9.

86 Zwart TC, Smits MG, Egberts TCG, et al. Long-term melatonin therapy for adolescents and young adults with chronic sleep onset insomnia and late melatonin onset: evaluation of sleep quality, Chronotype, and lifestyle factors compared to age-related randomly selected population cohorts. Healthcare 2018:6:e23 
87 Sadeghniiat-Haghighi K, Bahrami H, Aminian O, et al. Melatonin therapy in shift workers with difficulty falling asleep: a randomized, double-blind, placebo-controlled crossover field study. Work 2016:55:225-30.

88 Mostafavi A, Solhi M, Mohammadi M-R, et al. Melatonin decreases olanzapine induced metabolic side-effects in adolescents with bipolar disorder: a randomized double-blind placebo-controlled trial. Acta Med Iran 2014;52:734-9.

89 Calabrese JR, Guelfi JD, Perdrizet-Chevallier C, et al. Agomelatine adjunctive therapy for acute bipolar depression: preliminary open data. Bipolar Disord 2007;9:628-35.

90 Taylor D, Sparshat A, Varma S, et al. Antidepressant efficacy of agomelatine: meta-analysis of published and unpublished studies. BMJ 1888;2014.

91 Kennel J, Baus C, Dogui R, et al. Agomelatine-Related shift to mania in a patient with recurrent depressive disorder. Prim Care Companion
CNS Disord 201710.4088/PCC.16I02079. [Epub ahead of print: 26 Oct 2017].

92 Howland RH. A Benefit-risk assessment of agomelatine in the treatment of major depression. Drug Saf 2011;34:709-31.

93 Schnell A, Sandrelli F, Ranc V, et al. Mice lacking circadian clock components display different mood-related behaviors and do not respond uniformly to chronic lithium treatment. Chronobiol Int 2015;32:1075-89.

94 Moreira J, Geoffroy PA. Lithium and bipolar disorder: impacts from molecular to behavioural circadian rhythms. Chronobiol Int 2016;33:351-73.

95 Rock PL, Goodwin GM, Wulff K, et al. Effects of short-term quetiapine treatment on emotional processing, sleep and circadian rhythms. J Psychopharmacol 2016;30:273-82.

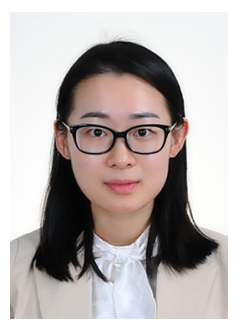

Yiming Chen obtained her bachelor degree from the Medicine School of Shanghai Jiao Tong University in 2017. She is now a PHD candidate of the medical school of Shanghai Jiao Tong University. Her research interests include bipolar disorder, sleep, feeding and eating disorders. 